\title{
Modelado predictivo de la epidemia de Chikungunya
}

Predictive modeling of the Chikungunya epidemic.

\section{Modèle prédictif de l'épidémie de Chikungunya}

\section{Modelagem preventiva da epidemia de Chikungunya}

Felipe Martínez T.*/ Geovanni Gil C.** Nikolai Guzmán G.***/ Tania Castañeda**** Nicolás Buitrago $* * * * *$ / Eder Bohórquez $* * * * * *$ Iván Mura $* * * * * * *$

Fecha de recepción: 6 de octubre

Fecha de aprobación: 28 de octubre

Pp. 7-32

* Estudiante Ingeniería Química, Universidad EAN.

** Estudiante Ingeniería Química, Universidad EAN.

*** Estudiante, Ingeniería de Energía, Universidad EAN

${ }^{* * * *}$ Estudiante de Ingeniería Química, Universidad EAN.

${ }^{* * * * *}$ Estudiante de Negocios Internacionales, Universidad EAN.

****** Estudiante de Ingeniería Química, Universidad EAN.

***** Ph.D. en Ingeniería Electrónica, Informática y de Telecomunicaciones, Universidad de Pisa. Magister en Ciencias de la Información, Universidad de Pisa y en Information Technology Project Management, George Washington University School of Business. 


\section{RESUMEN}

Este artículo presenta los resultados preliminares de una investigación multidisciplinaria que se está realizando en un proyecto de semillero en la Universidad EAN, y que tiene como objetivo el desarrollo de un modelo matemático predictivo de la difusión del Chikungunya en Colombia. Para enfrentarse a la epidemia, es necesario disponer de predicciones sobre la difusión de la enfermedad que permiten planear las respuestas sanitarias necesarias, así como evaluar la efectividad de las acciones de contraste, como por ejemplo la lucha química o biológica contra los mosquitos vectores. En el presente trabajo, se muestran los elementos esenciales para la construcción de modelos matemáticos basados en un sistema de ecuaciones diferenciales, cuya solución en el tiempo, proporcione una predicción a las poblaciones de humanos y vectores infectados por el virus del Chikungunya. El trabajo se fundamenta en las técnicas de modelado de sistemas biológicos, y a partir de una revisión de la literatura, propone construir un modelo que incluya los aspectos principales que afectan la difusión de la enfermedad y que se pueda adaptar a las condiciones específicas del área bajo objeto de estudio. El resultado final de la investigación, proporcionará una herramienta valiosa para implementar en la toma de decisiones en cuanto a las respuestas más adecuadas al momento de contrastar y controlar la difusión de esta enfermedad.

\section{Palabras CLAVe}

Biología, Chikungunya, modelado matemático, interdisciplinariedad.

\section{AbStRAct}

This article presents the preliminary results of a multidisciplinary research carried out on a project seedbed at the EAN University, which aims at developing a predictive mathematical model of the Chikungunya dissemination in Colombia. To address the epidemic, it is necessary to dispose of predictions about the dissemination of the disease that favor the planning of the necessary health responses as well as the evaluation of the effectiveness of contrasting actions, such as the chemical or biological control of mosquito vectors. This work presents the essential elements for the construction of mathematical models based on a system of differential equations, whose solution in time provide a prediction of human populations and vectors infected with the Chikungunya virus. This work is based on the modeling techniques of biological systems; and from a literature review, it 
aims at building a model that includes the main issues affecting the velocity of propagation of the disease that can be adapted to the specific conditions of the subject area under study. The final result of the research will provide a valuable tool to improve decision-making regarding the most suitable answers to contrast and control the dissemination of the Chikungunya.

\section{KEYWORDS}

Biology, Chikungunya, mathematical modeling, interdisciplinarity.

\section{RÉSUMÉ}

Cet article présente les résultats préliminaires d'une recherche pluridisciplinaire réalisée lors d'un projet de I'Université EAN ayant pour objectif de développer un modèle mathématique prédictif de la diffusion du Chikungunya en Colombie. Pour faire front cette l'épidémie, il est nécessaire de disposer de prédictions de diffusion de la maladie ce qui permet d'anticiper les réponses sanitaires nécessaires et d'évaluer l'effectivité des actions déjà entreprises au moyen de produits chimiques ou biologiques contre les moustiques porteurs du virus. Dans ce travail nous présentons les éléments fondamentaux à la mise en place de modèles mathématiques basés sur un système d'équations différentielles offrant une prédiction aux populations et une meilleure connaissance des vecteurs d'infection du virus du Chikungunya. L'analyse repose sur les techniques et modèles des systèmes biologiques, et grâce à une révision de la littérature disponible sur le sujet, nous proposons un modèle montrant les principaux aspects affectant la vitesse de diffusion de la maladie et pouvant s'adapter aux conditions spécifiques du domaine de cette étude. Le résultat final de la recherche fournira un outil précieux pour l'amélioration de la prise de décisions concernant les meilleures actions à prendre pour contrôler et arréter la diffusion du Chikungunya.

\section{MOTS CLEFS}

Biologie, Chikungunya, modèle mathématique, interdisciplinarité 


\section{RESUMO}

Este artigo apresenta os resultados preliminares de uma investigação multidisciplinar que se está realizando em um projeto da incubadora de projetos da Universidade EAN, que tem como objetivo desenvolver um modelo matemático de previsão da propagação do Chikungunya na Colômbia. Para enfrentar a epidemia, é necessário fazer previsões sobre a propagação da doença, que permitam planejar as respostas sanitárias necessárias assim como avaliar a eficácia das ações de contraste, como o controle químico ou biológico dos mosquitos vetores. Neste artigo se apresentam os elementos essenciais para a construção de modelos matemáticos, com base em um sistema de equações diferenciais, cuja solução a tempo venha a fornecer uma previsão das populações humanas e mosquitos vetores infectados com o vírus Chikungunya. $O$ trabalho baseia-se nas técnicas de modelagem de sistemas biológicos, e a partir de uma revisão da literatura, pretende construir um modelo que inclui as principais questões que afetam a rapidez da propagação da doença, e que possam adaptar as condições específicas da área de estudo. O resultado final da pesquisa irá fornecer uma ferramenta valiosa para melhorar a tomada de decisões sobre as respostas mais adequadas ao contraste e ao controle da propagação do Chikungunya.

\section{Palavras-chave}

Biologia, Chikungunya, modelagem matemática, interdisciplinares. 


\section{Introducción}

I virus del Chikungunya es un agente infectivo que utiliza

el mosquito como vector de transmisión. En tiempos recientes, esta epidemia se ha extendido de sus lugares nativos, las áreas tropicales de África, hacia Asia y América Latina (OPS, 2011). Al momento, no existe ninguna forma de tratamiento específico ni vacunas para la enfermedad, los únicos medios de control de la difusión siendo aquellos que contrastan la actividad del vector como agente transmisor. Sin embargo, la lucha a la enfermedad también requiere de la capacidad de generar predicciones sobre la difusión del virus, sea en condiciones de ausencia de control, sea cuando se esté evaluando la posible eficacia de medios de contraste a la transmisión, como cuarentena de los infectados o reducción de la población del vector.

Nuevas herramientas para buscar soluciones a diversos problemas en el área biológica, han venido apareciendo en los últimos años. Entre ellas, las técnicas de modelado, que suelen ser clasificadas como parte de la bioinformática o también de la biología de sistemas (Kitano, 2002), permiten generar predicciones sobre la evolución de sistemas complejos a través de formulaciones matemáticas de la dinámica de los sistemas que se analizan a través de la simulación.

La idea de aplicar las técnicas de modelado predictivo a la difusión de la enfermedad Chikungunya, define el proyecto de investigación multidisciplinario que se presenta en este artículo, y que está siendo desarrollado por los autores en el marco de un proyecto de semillero en la Universidad EAN. El objetivo final del proyecto es construir un modelo de la difusión de la enfermedad en Colombia, a través de la representación de las dinámicas de las poblaciones de los 
agentes vectores -mosquitos- y de los huéspedes -humanos-. Dicho trabajo de investigación requiere competencias de biología, de estadística y de sistemas; el equipo de proyecto está conformado por integrantes inscritos a diferentes facultades de la Universidad EAN.

La relevancia del tema es obvia, dado el estado de vulnerabilidad de la población y la necesidad de planear las respuestas reactivas -capacidad del sistema de salud-, y proactivas -control activo de la difusión-. Una señal de la importancia de las herramientas de modelado es visible en publicaciones derivadas de investigaciones y en revistas indexadas de gran importancia (Yakob \& Clements, 2013, Robinson \& otros, 2014). Así mismo, es importante resaltar la total incipiencia de la investigación del tema en Colombia: una búsqueda en la base de datos SCOPUS, arroja un total de 17 artículos publicados de autores colombianos que contienen la palabra clave Chikungunya hasta el 2015, de los cuales tres fueron publicados en 2014 y 14 en 2015.

El objetivo de este artículo es presentar los resultados preliminares que se obtuvieron en el proyecto de investigación que actualmente se está desarrollando en la Universidad EAN, como parte de las actividades del semillero IMANIN de la Facultad de Ingeniería. El proyecto pretende crear un modelo predictivo de la difusión del Chikungunya en el país, basado en el modelado continuo y determinístico de las poblaciones de humanos y mosquitos vectores. Las dinámicas de las variables del modelo serán representadas a través de un sistema de ecuaciones diferenciales ordinarias, que relacionan la evolución del número de humanos y de mosquitos infectados según el enfoque de modelado SEIR, comúnmente utilizado en epidemiología (Keeling \& Rohani, 2008). El modelo se construirá con la herramienta COPASI (Hoops \& otros, 2006), que proporciona una interfaz gráfica sencilla para la definición del mismo y realiza la integración de las ecuaciones 
diferenciales para obtener las predicciones de las dinámicas en el tiempo. En el momento en el cual se escribe este documento, el proyecto está en su etapa de diseño y creación de prototipos del modelo y de adaptación al caso específico colombiano; por ende, los resultados que se presentan son principalmente el marco teórico y técnico de la investigación, junto con las líneas de trabajo que se seguirán para completar los entregables.

El resto del artículo está estructurado como sigue: en el segundo apartado, se recapitulan, de manera concisa, la historia, los aspectos biológicos y aquellos médicos-epidemiológicos de la enfermedad. El tercer apartado introduce el modelado como herramienta para la predicción de la evolución temporal de sistemas biológicos, y describe una selección de artículos de la literatura que se enfrentan al mismo problema que se trata en este estudio. En el cuarto apartado, se presenta el enfoque de modelado SEIR, utilizando como ejemplo concreto uno de los estudios descritos en el tercer apartado. Finalmente, se presentan las conclusiones y extensiones que se aportarán a los modelos de la literatura, y que constituyen los elementos del futuro trabajo del proyecto de semillero. 


\section{La enfermedad Chikungunya}

n este apartado se presenta un resumen de los datos - y la información disponible sobre la difusión del Chikungunya, los aspectos biológicos de la transmisión del virus y patológicos que este determina.

\subsection{Difusión del Chikungunya}

El virus del Chikungunya fue descrito por primera vez hacia el año de 1955 por Robinson Marion en ocasión de un brote epidémico en Tanzania, siendo el virus autóctono del África Ssubsahariana donde se presenta un ciclo selvático entre mosquitos y primates (Robinson, 1955). Según la OMS, después de la identificación del virus responsable de la enfermedad, que fue nombrado CHIKV, apareció en el 2004 un brote esporádico en Kenia, que se transmitió y afectó lugares cercanos como Coromoro, La Reunión, y otras islas del océano Índico. Entre el 2004 y el 2006 hubo aproximadamente 500.000 casos de infección (OPS, 2011).

A partir de 2006 la epidemia se extendió a la India infectando a 1,39 millones de personas en sus 17 de 28 Estados, y desde la India el virus llegó a las islas Andamán y Nicobar, Sri Lanka, las Maldivas, Singapur, Malasia e Indonesia, transmitido por viajeros infectados.

Las alertas se dispararon en el año 2007 cuando se descubrió que el virus se estaba transmitiendo de manera autóctona en el norte de Italia. En el periodo entre 2006 al 2011, se reportaron 117 casos de fiebre Chikungunya entre ciudadanos de los Estados Unidos, todos derivados de 
infecciones adquiridas en viajes a áreas con brotes en curso conocidos.

En 2013, la OMS declaró el ingreso de la enfermedad en las Américas y en las islas del Caribe, y la confirmación de la transmisión autóctona del virus. Sin embargo, el virus se mantiene activo en el continente por la presencia de mosquitos capaces de transmitir la enfermedad, así como de huéspedes susceptibles.

En agosto de 2014, el Instituto Nacional de Salud de Colombia emanaba el Plan Nacional de Respuesta Frente a la Introducción del Virus Chikungunya en Colombia (INS, 2014), anunciando que no existían, a la fecha, casos documentados de transmisión autóctona de la enfermedad. En abril de 2015, según informa el Instituto Nacional de Salud, la enfermedad se transmite de manera autóctona en todo el territorio nacional; lo casos confirmados suman más de 180.000 y se reportan 45 casos de muerte debido a la enfermedad (INS, 2015).

\subsection{Aspectos biológicos de la enfermedad}

La fiebre del Chikungunya es causada por un alphavirus, cuyo material genético consiste en una cadena de ARN simple que no necesita de ADN para replicarse. El virus es transmitido a través de la picadura de las hembras de mosquitos Aedes aegypti -el común mosquito hogareño-.

El virus CHIKV se reproduce inicialmente en el sitio de la picadura y se difunde después en todo el organismo (Tang, 2012). El virus es capaz de infectar varios tipos de células incluyendo las epiteliales, las nerviosas, las musculares y los macrófagos, siendo los fibroblastos las células donde la replicación viral es más eficiente (Sourisseau \& otros, 2007). 
La respuesta del organismo humano a la infección, está inicialmente basada en la producción de interferón de tipo I. Esta respuesta es suficiente para eliminar la infección, lo que comúnmente ocurre antes de que el organismo empiece a producir anticuerpos específicos (Schilte \& otros, 2010).

La enfermedad del Chikungunya solo se transmite a través de la picadura del mosquito después que este haya ingerido sangre de un humano infectado. Normalmente, la incubación del virus en el mosquito tiene un periodo promedio de diez días; después de este tiempo el mosquito es capaz de transmitir la enfermedad. El ciclo vital de los vectores es muy importante para entender los factores que afectan la rapidez del contagio.

Aedes aegypti es un mosquito que mide menos de un centímetro, y por volar sin emitir sonido, es un vector muy eficaz en la transmisión de enfermedades entre los humanos. Este mosquito cuenta con hábitos de vida adaptados a explotar las oportunidades ofrecidas por las instalaciones humanas. Los huevos son depuestos -alrededor de 150-200 por deposición- en cualquier recipiente con agua estancada, y las larvas nacen entre dos y siete días después, dependiendo de la temperatura del agua. En un periodo mínimo de cuatro días, las larvas se desarrollan a través de un proceso que pasa por cuatro estadios hasta convertirse en pupas. Bajo condiciones no aptas de temperatura, las larvas pueden tardar en su desarrollo hasta meses. De las pupas, en pocos días, emergen nuevos insectos adultos; estos últimos viven aproximadamente entre dos y cinco semanas, dependiendo de las condiciones ambientales. Por su parte, las hembras alcanzan a reproducirse hasta cinco veces en su vida.

Según reportan Staples y otros (2009), nuevas investigaciones han identificado una mutación del virus que le permite transmitirse a través de la picadura del Aedes 
albopictus, vulgarmente conocido como el mosquito tigre. Ese insecto, originario de Asia, es una de las especies más invasivas, tiene hábitos de vida similares al Aedes aegypti, y en años recientes ha venido colonizando todas la áreas tropicales y templadas del planeta (Benedict \& otros, 2007). Ambas especies de mosquitos son de amplia difusión en Colombia, en todas las regiones de altura inferior a los 2.200 metros sobre el nivel del mar, y son también responsables de la difusión del dengue. Estos insectos pueden picar durante todo el día, aunque el máximo de su actividad se encuentre al inicio de la mañana y al final de la tarde. Aedes aegypti se destaca por su aptitud a picar en ambientes interiores tanto como al aire libre.

\subsection{Aspectos de la patología viral CHIKV}

La fiebre de Chikungunya se caracteriza por un fuerte dolor articular y fiebre alta (39-40 grados). El mismo nombre de la enfermedad, Chikungunya, es una voz del idioma Kimakonde que significa doblarse, en alusión al aspecto encorvado de los pacientes debido a los dolores articulares. Además, produce otros síntomas, tales como dolores musculares, dolores de cabeza, náuseas, cansancio y erupciones cutáneas. Algunos de estos síntomas llevan a que la enfermedad sea confundida con el dengue.

Normalmente, la incubación del virus en los seres humanos varía entre tres y siete días, después de este tiempo inician los síntomas de la enfermedad. Estos perduran por poco tiempo, en particular la fiebre baja entre dos a tres días, mientras que los otros pueden durar más. Se han llegado a conocer casos en los que los síntomas perduran 10, 18 meses y hasta dos años -fuerte dolor articular-. El hecho de que el virus sea capaz de infectar los macrófagos y entonces eludir las defensas de las respuestas inmunológicas, podría ser la base de las recurrencias de los síntomas que se observan en estos casos. 
El virus puede diagnosticarse durante las primeras 48 horas a través de un análisis de sangre. La técnica ELISA -acrónimo del inglés Enzyme-Linked ImmunoSorbent Assay-, detecta los anticuerpos producidos por el sistema inmunológico como defensa ante el virus.

Según lo indica un texto publicado por la Organización Panamericana de la Salud (OPS) denominado <<Preparación y respuesta ante la eventual introducción del virus Chikungunya en las Américas $>>$ (2011), la enfermedad se considera en tres formas clínicas: aguda, subaguda y crónica, dependiendo de la gravedad de los síntomas. También, asegura que la enfermedad tiene una tasa de mortalidad de $0,4 \%$ para menores de un año y aumenta en los mayores de edad -especialmente en los mayores de 75 años- o en las personas que han sufrido de infecciones relacionadas.

Según indican los autores Pérez Sánchez \& otros (2014), las mujeres embarazadas y los jóvenes tienden a sufrir menor gravedad en los síntomas, y el tiempo de afección es menor con relación a otro tipo de pacientes. No se han reportado a la fecha casos de transmisión trans-placentaria, pero se han dado algunos casos de aborto. Actualmente se cree que una vez expuestos al CHIKV, los individuos desarrollan inmunidad prolongada que los protege contra la reinfección (OPS, 2011). 


\section{Modelado predictivo de sistemas biológicos}

a palabra modelo, es quizás una de las más utilizadas en la ciencia y toma un significado diferente según el ámbito y el contexto que se está utilizando. Incluso, cuando los seres humanos se enmarcan dentro de los límites de la biología, la ciencia de los sistemas vivos, el término modelo recuerda muchos significados diferentes. Por ejemplo, el dominio de la unión del hierro de la hemoglobina EME, es un caso de un modelo de estructura terciaria de proteínas; la común levadura Saccharomyces cerevisiae es un modelo, al igual que muchos otros organismos que se seleccionaron como sujetos de estudio para otras especies que son más difíciles de estudiar directamente. El mecanismo de acción de un fármaco, por ejemplo la aspirina, es un modelo para el mecanismo de la acción de compuestos no esteroides antiinflamatorios.

Se considera que un modelo es una generalización o abstracción de algún fenómeno o sistema utilizado para transmitir información cualitativa y/o cuantitativa, sobre el fenómeno/sistema que representa. Para ser más preciso habrá que considerar los modelos de los sistemas vivos que poseen las siguientes propiedades:

- Incluyen los aspectos cualitativos y cuantitativos de los sistemas vivos.

- Se pueden utilizar para determinar la evolución dinámica de un fenómeno/sistema con el tiempo, a partir de una condición conocida -estado inicial-. 
- Son susceptibles de simulación en un ordenador, lo que implica que tienen una solo semántica, es decir, un significado inequívoco.

La definición y simulación de modelos permite estudiar su evolución a través de la computación. La ventaja de usar modelos computacionales es múltiple:

- Pueden ser construidos a diferentes niveles de abstracción, lo que permite centrarse en el verdadero objeto de interés del estudio o investigación.

- Permiten explorar fenómenos de otro modo difícil o imposible de observar.

- Los estudios basados en modelos computacionales consumen mucho menos recursos -tiempo, dinero- que los experimentos de laboratorio.

Los puntos anteriores no suponen que los modelos puedan reemplazar a la experimentación biológica; más bien, proporcionan herramientas eficaces que complementan y ayudan a la investigación en biología; sostienen o refutan las hipótesis experimentales; e impulsan el diseño de experimentos.

Existen diferentes clases de modelado predictivo que son aplicados al estudio de sistemas biológicos (Mura, 2013). Entre ellos podemos mencionar los continuos-deterministas (Twycross et al., 2010), y aquellos discretos-estocásticos (Mura, 2010). En el contexto de este trabajo de investigación, nos limitamos a considerar el enfoque continuo-determinista, el más utilizado a la hora de construir modelos matemáticos en epidemiología. 
El proceso de definición de un modelo computacional susceptible de simulación, requiere de extraer, a través de un proceso de abstracción y medición, dos distintos tipos de información:

Información estructural, que incluye las entidades del sistema, sus posibilidades de evolución e interacción; esta información es normalmente visualizada en forma de una red de nodos (entidades) y sus interacciones.

Información cuantitativa, que proporciona los detalles acerca de la rapidez con que las entidades del sistema cambian su estado e interactúan, así como la multiplicidad de las entidades en el estado inicial del sistema.

El modelado predictivo de sistemas biológicos se ha aplicado a todos las escalas de los sistemas vivos, de las redes de control de la transcripción genética (Ihekwaba et al., 2014), a las interacciones entre células en un organismo (Csikász-Nagy et al., 2012), hasta llegar a enteros ecosistemas (Scotti et al., 2012).

En el contexto del modelado de la epidemia de Chikungunya, se encuentran en la literatura varios trabajos. Entre los más recientes mencionamos Moulay \& otros (2012), donde los autores utilizan un modelo de las dinámicas poblacionales basado en ecuaciones diferenciales para determinar las acciones de control del vector que tienen el mejor resultados en cuanto a la reducción de la velocidad de difusión de la enfermedad. Yakob y Clements (2013), utilizan un modelo basado en ecuaciones diferenciales y los datos de un brote de Chikungunya en la isla Reunión, para estudiar la capacidad del modelo de reproducir las dinámicas observadas de la difusión. En (Robinson et al., 2014), un modelo discreto estocástico es construido a partir de los datos de un brote en Camboya, y analizado para determinar la sensibilidad 
con respecto a la precisión en la medición del número de infectados. Por último, el muy reciente trabajo de Alkama et al. (2015), se enfrenta al reto determinar la estrategia optima de control de la epidemia, con base en el análisis de un modelo matemático a tiempo discreto -ecuaciones de diferencias- de las poblaciones de humanos y vectores.

\section{Modelado de la difusión epidémica de la enfermedad}

n esta sección del documento presentamos las ideas de base del modelado, con referencia al trabajo de Yakob \& Clements (2013), en el cual los autores plantean un modelo de tipo SEIR de las dinámicas de la epidemia en la isla de Reunión.

Los modelos de tipos SEIR son modelos donde la población del huésped (en este caso el humano), es dividida en cuatro grupos separados:

- Grupo S -Susceptible, en Ingles-, donde se agrupan los individuos susceptibles de enfermarse.

- Grupo E -Exposed, en Ingles-, que agrupa los individuos que han sido expuestos al contagio pero están todavía enfermos.

- Grupo I-Infected, en Ingles-, la sub-población de infectados.

- Grupo R -Recovered, en Ingles-, de los individuos que han desarrollado la inmunidad a la enfermedad. 
En Yakob \& Clements (2013), el grupo I se considera dividido de hecho en dos sub-grupos, para tener en cuenta los infectados que desarrollan la enfermedad de manera asintomática (sub-grupo la), y sintomática (sub-grupo I).

En cuanto a la población del vector, esta queda también repartida en tres grupos, los mosquitos susceptibles (grupo $\mathrm{X}$ ), los expuestos (grupo Y) y los infectados (grupo Z) (Figura 1).

Figura 1. Dinámicas inter-grupales en el modelo SEIR.

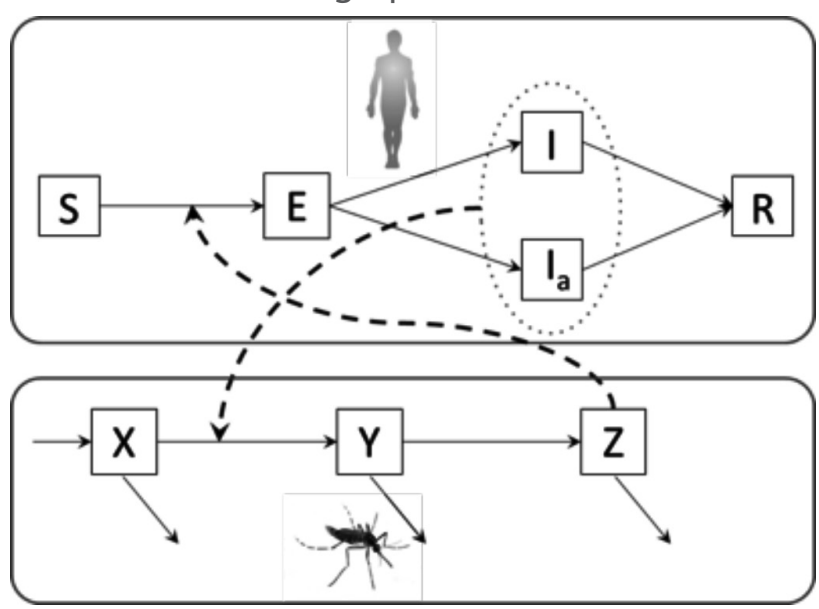

Fuente. Elaboración propia a partir de Yakob \& Clements, (2013).

Las dinámicas entre grupos, que se ilustran en la Fuente, recapitulan la información estructural del modelo, es decir las entidades (los grupos, en este caso), y las interacciones entre ellos. Las flechas continuas representan los cambios de grupo de los individuos en las poblaciones, y las flechas discontinuas las interacciones entre poblaciones diferentes.

El modelo descrito anteriormente, desde luego necesita ser completado con la información cuantitativa, es decir, en número de individuos en cada grupo en el estado inicial y las tasas que definen la velocidad o frecuencia de las interacciones. 
Utilizando el software COPASI (Hoops et al., 2006), es posible codificar las interacciones representadas, en un lenguaje inspirado en las reacciones bioquímicas, que de manera simple permite construir el modelo sin necesidad de lidiar con su representación matemática en forma de sistema de ecuaciones diferenciales. Las reacciones que definen el modelo SEIR presentado en forma diagramática se presentan a continuación (Tabla 1).

Tabla 1. Reacciones para el modelo SEIR.

\begin{tabular}{|c|c|c|}
\hline$\#$ & Reacción & Descripción \\
\hline 1 & $S+Z->E+Z$ & $\begin{array}{l}\text { Los susceptibles son expuestos al virus por la } \\
\text { picadura de un mosquito infectado }\end{array}$ \\
\hline 2 & $E->I$ & $\begin{array}{l}\text { Los expuestos desarrollan el contagio, de forma } \\
\text { sintomática }\end{array}$ \\
\hline 3 & E $\rightarrow$ la & $\begin{array}{l}\text { Los expuestos desarrollan el contagio, de forma } \\
\text { asintomática }\end{array}$ \\
\hline 4 & $I->R$ & $\begin{array}{l}\text { Los infectados de forma sintomática desarrollan } \\
\text { la inmunidad }\end{array}$ \\
\hline 5 & la $->$ R & $\begin{array}{l}\text { Los infectados de forma asintomática desarrollan } \\
\text { la inmunidad }\end{array}$ \\
\hline 6 & $\varnothing->x$ & Nacimiento de los mosquitos \\
\hline 7 & $X+I->Y+I$ & $\begin{array}{l}\text { Los mosquitos son expuestos al virus por picar un } \\
\text { humano infectado de forma sintomática }\end{array}$ \\
\hline 8 & $\begin{array}{l}X+l a->Y+ \\
l a\end{array}$ & $\begin{array}{l}\text { Los mosquitos son expuestos al virus por picar un } \\
\text { humano infectado de forma asintomática }\end{array}$ \\
\hline 9 & $Y \rightarrow Z$ & $\begin{array}{l}\text { Los mosquitos expuestos desarrollan la } \\
\text { enfermedad }\end{array}$ \\
\hline 10 & $X->\varnothing$ & $\begin{array}{l}\text { Muerte de los mosquitos no expuestos ni } \\
\text { infecciosos }\end{array}$ \\
\hline 11 & $Y \rightarrow \varnothing$ & Muerte de los mosquitos expuestos \\
\hline 12 & $Z->\varnothing$ & Muerte de los mosquitos infecciosos \\
\hline
\end{tabular}

Fuente. Yakob \& Clements, (2013). 
Según las reacciones recién presentadas, la población de los humanos se asume constante, es decir no se modelan variaciones en la población total suma de los compartimientos S, E, I y R por nacimientos o muertes, mientras se considera la variación de la población del vector, que tiene una dinámica mucho más rápida.

Utilizando la información cuantitativa reportada en Yakob \& Celements (2013), es posible determinar las tasas o velocidades de las reacciones que se enumeraron. Esta información cuantitativa es reportada más adelante (Tabla 2).

Tabla 2. Tasas de reacción para las reacciones del modelo SEIR.

\begin{tabular}{|c|c|c|c|c|}
\hline$\#$ & Reacción & $\begin{array}{l}\text { Tasa de } \\
\text { reacción }\end{array}$ & Valor & Unidades \\
\hline 1 & $S+Z>E+Z$ & $\mathrm{k}_{1}$ & 0.0005 & unidades* días ${ }^{-1}$ \\
\hline 2 & $E>I$ & $\mathrm{k}_{2}$ & 0.225 & días $^{-1}$ \\
\hline 3 & $E>I_{a}$ & $k_{3}$ & 0.05 & días $^{-1}$ \\
\hline 4 & $\mathrm{I}>\mathrm{R}$ & $k_{4}$ & 0.25 & días $^{-1}$ \\
\hline 5 & $\mathrm{I}_{a}>\mathrm{R}$ & $k_{5}$ & 0.25 & días $^{-1}$ \\
\hline 6 & $\varnothing>x$ & $\mathrm{k}_{6}$ & 20000 & (unidades* días \\
\hline 7 & $X+1>Y+I$ & $\mathrm{k}_{7}$ & 0.00005 & unidades $^{*}$ días $^{-1}$ \\
\hline 8 & $X+I_{a}>Y+I_{a}$ & $\mathrm{k}_{8}$ & 0.00005 & unidades* días $^{-1}$ \\
\hline 9 & $Y>Z$ & $k_{9}$ & 0.25 & días $^{-1}$ \\
\hline 10 & $x>\varnothing$ & $k_{10}$ & 0.04 & días $^{-1}$ \\
\hline 11 & $Y>\varnothing$ & $k_{11}$ & 0.04 & días $^{-1}$ \\
\hline 12 & $Z>\varnothing$ & $\mathrm{k}_{12}$ & 0.04 & días $^{-1}$ \\
\hline
\end{tabular}

Fuente. Yakob \& Clements, (2013).

La combinación de la información cualitativa proporcionada en la Tabla 1 y aquella cuantitativa que se reporta en la Tabla 2 permite obtener el siguiente modelo matemático en forma de ecuaciones diferenciales ordinarias acopladas: 


$$
\left\{\begin{array}{lcc}
\frac{d}{d t} S(t)= & -k_{1} \cdot S(t) \cdot Z(t) \\
\frac{d}{d t} E(t)= & k_{1} \cdot S(t) \cdot Z(t)-k_{2} \cdot E(t)-k_{3} \cdot E(t) \\
\frac{d}{d t} I(t)= & k_{2} \cdot E(t)-k_{4} \cdot I(t) \\
\frac{d}{d t} I_{a}(t)= & k_{3} \cdot E(t)-k_{5} \cdot I_{a}(t) \\
\frac{d}{d t} R(t)= & k_{4} \cdot I(t)+k_{5} \cdot I_{a}(t) \\
\frac{d}{d t} X(t)= & k_{6}-k_{7} \cdot X(t) \cdot I(t)-k_{8} \cdot X(t) \cdot I_{a}(t)-k_{10} \cdot X(t) \\
\frac{d}{d t} Y(t)= & k_{7} \cdot X(t) \cdot I(t)+k_{8} \cdot X(t) \cdot I_{a}(t)-k_{11} \cdot Y(t) \\
\frac{d}{d t} Z(t)= & k_{9} \cdot Y(t)-k_{12} \cdot Z(t)
\end{array}\right.
$$

En el modelo matemático, una variable continua es introducida por cada compartimiento del modelo SEIR. Por ejemplo, la variable continua $\mathrm{S}(\mathrm{t})$ representa en el modelo el número de humanos susceptibles en cada momento en la evolución del sistema. Este mismo modelo es construido de manera automática a través de la herramienta COPASI, que solo necesita ingresar la información recopilada en la Tabla 1 y la Tabla 2. Una vez ingresada la información, COPASI permite realizar una simulación del modelo para determinar cómo evolucionaran las poblaciones de mosquitos y humanos a partir de un estado inicial. Dicha simulación es realizada a través de la integración numérica del sistema de ecuaciones diferenciales.

Realizamos una simulación numérica del modelo a partir de un estado inicial donde solo un humano es infectado en la población (de 50,000 unidades totales), es decir, la numerosidad del grupo I es 1, y todos los demás están en el grupo $\mathrm{S}$, y todos los mosquitos son susceptibles -grupo $X-$, numerosidad 500,000 unidades). Se reporta la evolución simulada en el intervalo temporal $[0,50]$ días de la población de los mosquitos, en los tres grupos $\mathrm{X}, \mathrm{Y}$ y $\mathrm{Z}$ (Figura 2). La simulación permite determinar la difusión del virus en la población del mosquito y determina el pico máximo del peligro de infección alrededor del día 18 -valor máximo predicho para el número de mosquitos infecciosos, grupo Z-. 
Figura 2. Evolución simulada de la infección en la población de mosquitos.

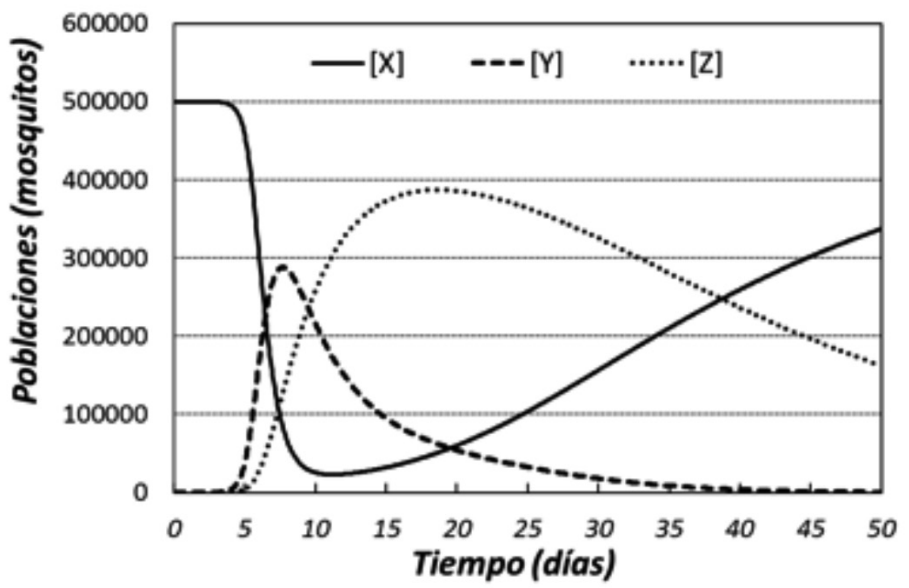

Fuente. Elaboración propia de los autores.

Las predicciones para los humanos son reportadas a continuación (Figura 3). En las curvas simuladas a la derecha, es posible determinar que el máximo número de casos se presentará alrededor del día diez del inicio del contagio.

Figura 3. Evolución simulada de la infección en la población humana.
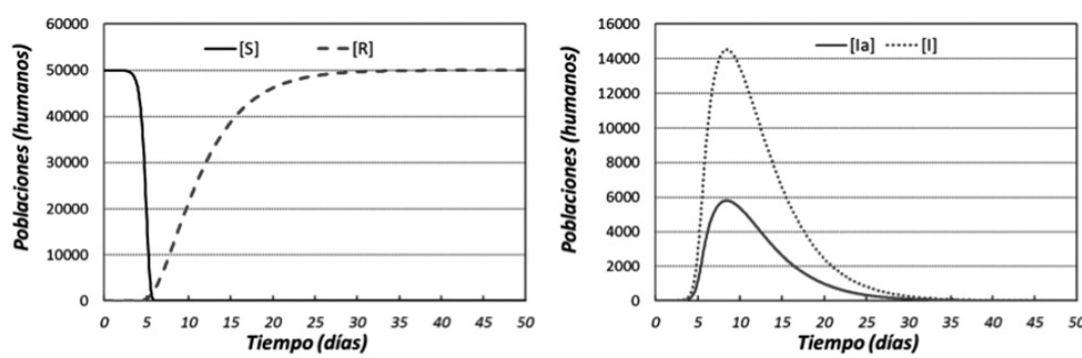

Fuente. Elaboración propia de los autores.

Las proporcionan la predicción del número de infectados en el tiempo, la información más importante para la planeación de las respuesta sanitarias. 


\section{Conclusiones}

- I modelado matemático de las dinámicas poblacionales de huéspedes y vectores, proporciona una herramienta valiosa para determinar predicciones de la difusión de la epidemia del Chikungunya. Existen aplicativos software que permiten construir y simular modelos complejos de manera muy sencilla, así que se hace posible realizar estudios detallados que incluyen información puntual sobre varios aspectos que afectan las dinámicas del modelo. En este artículo, reportamos el resultado de un estudio preliminar que apunta a determinar las posibilidades de construir modelos matemáticos predictivos para la difusión de la epidemia en Colombia, y presentamos técnicas y herramientas que, sin requerir conocimientos especializados en modelaje matemático, permiten representar las evoluciones en el tiempo de las poblaciones de vectores y de huéspedes humanos.

El modelo que se presenta es relativo a un brote de la epidemia de Chikungunya que afecto a la islas Reunión, y que fue estudiado en el trabajo de Yakob y Celements (2013). La adaptación de este modelo a la realidad colombiana requiere primariamente re-estimar las tasas que definen el ciclo de vida del mosquito, que dependen de factores climáticos y ambientales de la zona donde se propaga la enfermedad. Así mismo, es necesario evaluar las tasas de contagio, que son determinadas por aspectos socioeconómicos tales como la actividad laboral de la población y el grado de difusión de elementos que protegen de las picaduras en las viviendas.

Varias extensiones del modelo SEIR que se revisa en este artículo son también posibles y sencillas por implementar y simular en la herramienta COPASI. Por ejemplo, la diferente susceptibilidad por edades, o la estacionalidad de las Iluvias. 
Estos factores serán considerados en el trabajo futuro del equipo del proyecto del semillero, para definir un modelo que sea representativo de las áreas geográficas que serán objeto del estudio.

\section{Referencias bibliográficas}

Alkama, M., Rachik, M., \& otros. (2015), Optimal Control Strategy for a Discrete Chikungunya Model, International Journal of Science and Research, 4: 3, pp. 398-404.

Benedict, M.Q., Levine, \& otros, L.P. (2007). Spread of the Tiger: Global Risk of Invasion by the Mosquito Aedes albopictus. Vector Borne and Zoonotic Diseases (Larchmont, N.Y.), 7(1), pp. 76-85. doi:10.1089/vbz.2006.0562.

Csikász-Nagy, A., Cavaliere, M., \& otros. (2012). Combining Game Theory and Graph Theory to Model Interactions between Cells in the Tumor Microenvironment. In A. d'Onofrio, P. Cerrai, \& A. Gandolfi (Eds.), New Challenges for Cancer Systems Biomedicine: Part 1. (pp. 3-18). Springer.

Erin Staples J., Breiman R.F., \& otros. (2009), Chikungunya Fever: An Epidemiological Review of a Re-Emerging Infectious Disease, Clin Infect Dis. (2009) 49 (6), pp. 942948, doi:10.1086/605496.

Ihekwaba, A., Mura, I., \& otros, (2014). Computational modelling and analysis of the molecular network regulating sporulation initiation in Bacillus subtilis, BMC Systems Biology 8:119, doi:10.1186/s12918-014-0119-x.

INS - Instituto Nacional de Salud de Colombia (2014). Plan Nacional de Respuesta Frente a la Introducción del Virus 
Chikungunya en Colombia. Recuperado de http://www. minsalud.gov.col

. (2015). Lo que debes saber sobre la fiebre Chicunguña. Recuperado de http://www.ins.gov.co/

Hoops, S., Sahle, S., \& otros. (2006). COPASI: a COmplex PAthway SImulator, Bioinformatics, 22, pp. 3067-3074.

Kitano, H., (2002). Systems Biology: a brief overview, Science, 195, pp. 1662-1664, doi:10.1126/science. 1069492

Keeling, M.J. \& Rohani, P. (2008). Modeling Infectious Diseases: in Humans and Animals. Princeton, Princeton University Press.

Moulay, D., Aziz-Alaoui, M.A., \& otros. (2012), Optimal Control of Chikungunya Disease: Larvae Reduction, Treatment and Prevention, Mathematical Biosciences and Engineering 9:2, pp. 369-393, doi:10.3934/mbe.2012.9.369

Mura, I. (2010). Stochastic Modeling, in I. Koch, W. Reisig and F. Schreiber (eds.), Modeling in Systems Biology, The Petri Net Approach, Computational Biology 16. SpringerVerlag London Limited, chapter 7.

(2013). On Modeling Approaches for the Predictive Simulation of Living Systems Dynamics, ONTARE, Revista de investigación Facultad de Ingeniería EAN, Vol. 1, num.2 pp. 101-124.

OPS-Organización Panamericana de la Salud. (2011), Preparación y respuesta ante la eventual introducción del virus Chikungunya en las Américas. Recuperado de http:// www1.paho.org.

Pérez Sánchez, G., Ramírez Alvarez, G., \& otros. (2014), Fiebre de Chikungunya: enfermedad infrecuente como emergencia médica en Cuba, MEDISAN; 18(6), pp. 848-856. 
Robinson, M.C. (1955) An epidemic of virus disease in Southern Province, Tanganyika territory, in 1952-1953. Trans R Soc Trop Med Hyg 49, pp. 28-32.

Robinson, M., Conan, A., \& otros. (2014). A model for a chikungunya outbreak in a rural Cambodian setting: implications for disease control in uninfected areas, PLOS Negl Trop Dis. 8:9, doi: 10.1371/journal.pntd.0003120

Schilte, C., Couderc, T., Chretien, \& otros. (2010). Type I IFN controls chikungunya virus via its action on nonhematopoietic cells. J Exp Med 207(2), pp. 429-442, doi:10.1084/jem.20090851.

Scotti, M., Gjata, N., Livi, C., \& otros, (2012). Dynamical effects of weak trophic interactions in a stochastic food web simulation, Community Ecology, 13, pp. 230-237.

Sourisseau, M., Schilte, \& otros. (2007). Characterization of reemerging chikungunya virus. PLoS Pathogens, 3, doi:10.1371/journal.ppat.0030089.

Tang, B.L. (2012). The cell biology of Chikungunya virus infection, Cellular Microbiology 14(9), pp. 1354-1363, doi:10.1111/j.1462-5822.2012.01825.x

Twycross, J., Band, L.R., \& otros, (2010). Stochastic and deterministic multiscale models for systems biology: an auxin-transport case study, BMC Systems Biology, 4, doi:10.1186/1752-0509-4-34

Yakob, L., \& Clements A.C.A. (2013). A Mathematical Model of Chikungunya Dynamics and Control: The Major Epidemic on Reunion Island, PLOS ONE 8(3), doi:10.1371/journal. pone.0057448. 